\title{
Partial Feedback Linearization of Underactuated Mechanical Systems *
}

\author{
Mark W. Spong \\ Coordinated Science Laboratory \\ University of Illinois at Urbana-Champaign \\ 1308 W. Main St., Urbana, IL 61801
}

\begin{abstract}
In this paper we discuss the partial feedback linearization control of underactuated mechanical systems. We consider an $n$ degree of freedom system having $m$ actuated, or active, degrees of freedom and $\ell=n-m$ unactuated, or passive, degrees of freedom. It is known that the portion of the dynamics corresponding to the active degrees of freedom may be linearized by nonlinear feedback. In this paper we show, alternatively, that the portion of the dynamics corresponding to the passive degrees of freedom may be linearized by nonlinear feedback under a condition that we call Strong Inertial Coupling. We derive and analyze the resulting zero dynamics which are crucial to an understanding of the response of the overall system. Simulation results are presented showing the performance of two link underactuated robots under partial feedback linearization control.
\end{abstract}

\section{Introduction}

Underactuated mechanical systems are mechanical systems with fewer actuators than degrees-of-freedom and arise in several ways, from intentional design as in the brachiation robot of Fukuda [13] or the Acrobot [2], in mobile robot systems when a manipulator arm is attached to a mobile platform, a space platform, or an undersea vehicle, [8], or because of the mathematical model used for control design as when joint flexibility is included in the model [14]. In the latter sense, then, all mechanical systems are underactuated if one wishes to control flexible modes that are not directly actuated (the noncollocation problem), or even to include such

*This research is partially supported by the National Science Foundation under grants MSS-9100618, INT-9202168, and IRI9216428 . things as actuator dynamics in the model description.

Our main interest in this paper is the control of gymnast type robots like the Acrobot [2], and the three-link gymnast robot in [17]. We will show that the method of partial feedback linearization [7] and the recent method of integrator backstepping [9] provide effective design tools for controlling such robots to perform various motions.

It has long been known [16] that fully actuated robots are feedback linearizable by nonlinear feedback. For underactuated robots it is known that the portion of the dynamics corresponding to the actuated (or active) degrees of freedom may be linearized by nonlinear feedback[5]. The remaining portion of the dynamics after such partial feedback linearization is nonlinear and represents internal dynamics. In this paper we show that, under a condition which we call Strong Inertial Coupling, it is alternatively possible to linearize the portion of the dynamics corresponding to nonactuated (or passive) degrees of freedom. This somewhat surprising result is quite interesting and, roughly speaking, means for a system with $m$ actuators, that $m$ of the equations of motion may be linearized whether or not they are directly actuated. We will show how these results may be used to control underactuated robots performing gymnastic type motions.

\subsection{Upper Actuated and Lower Actuated Systems}

We consider an $n$-degree-of-freedom system with generalized coordinates $q^{1}, \ldots, q^{n}$, and $m<n$ actuators, each of which directly actuates a single degree of freedom. Each actuated degree of freedom is called an active joint. The remaining $\ell=n-m$ unactuated degrees of freedom are called passive joints, as shown in Figure 1.

A so-called Upper Actuated System is one in which 


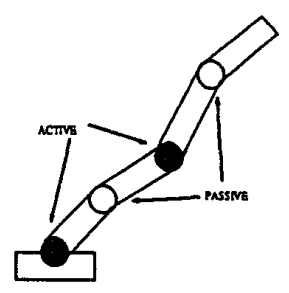

Figure 1: A General Underactuated System

the upper arm, or the first $m$-joints are actuated while a Lower Actuated System is one in which the lower arm, or the last $m$-joints are actuated (see Figure 2). By suitably numbering and partitioning the vector $q$ of generalized coordinates we may write $q^{T}=\left(q_{1}^{T}, q_{2}^{T}\right)$ where $q_{1} \in R^{\ell}$ corresponds to the passive joints and $q_{2} \in R^{m}$ corresponds to the active joints. Thus all systems will be considered as though they are lower actuated without loss of generality.

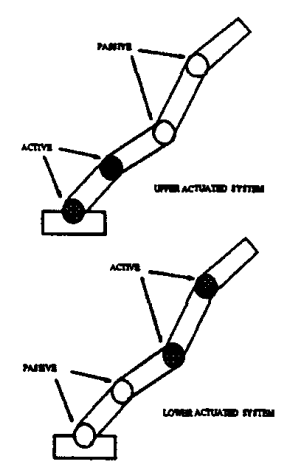

Figure 2: Upper and Lower Actuated Systems

\subsection{Dynamics}

With the vector $q \in R^{n}$ of generalized coordinates partitioned as above with $q_{1} \in R^{\ell}$ and $q_{2} \in R^{m}$, we may write the dynamic equations of the $n$ degree of freedom system as

$$
\begin{aligned}
& M_{11} \ddot{q}_{1}+M_{12} \ddot{q}_{2}+h_{1}+\phi_{1}=0 \\
& M_{21} \ddot{q}_{1}+M_{22} \ddot{q}_{2}+h_{2}+\phi_{2}=\tau
\end{aligned}
$$

where

$$
M(q)=\left[\begin{array}{ll}
M_{11} & M_{12} \\
M_{21} & M_{22}
\end{array}\right]
$$

is the symmetric, positive definite inertia matrix, the vector functions $h_{1}(q, \dot{q}) \in R^{\ell}$ and $h_{2}(q, \dot{q}) \in R^{m}$ contain Coriolis and centrifugal terms, the vector functions $\phi_{1}(q) \in R^{\ell}$ and $\phi_{2}(q) \in R^{m}$ contain gravitational terms, and $\tau \in R^{m}$ represents the input generalized force produced by the $m$ actuators at the active joints. For notational simplicity we will henceforth not write the explicit dependence on $q$ of these coefficients. The equations (1)-(2) represent the standard dynamics of $n$ link robots except that there is no control input to the first $\ell$ equations [16].

\section{Partial Feedback Linearization}

In this section we consider the Input/Output (or Partial) Feedback Linearization [7] of the system (1)(2). We first consider an output equation

$$
y_{2}=q_{2} \in R^{m} \text {. }
$$

In this case the output $y_{2}$ is collocated with the input $\tau$, i.e., with the active joints, and we recover the known results on input/output linearization for underactuated systems. Our contribution here is in the characterization of the resulting internal or zero dynamics [7] which will be important later in the application to the swing up control of the Acrobot.

Next we consider the input/output linearization relative to an output equation

$$
y_{1}=q_{1} \in R^{\ell} .
$$

In this case the output corresponds to the passive joints and is not collocated with the input. We show that input/output linearization is possible in this case under a condition that we call Strong Inertial Coupling. This condition allows the integrator backstepping formalism [9] to be used to linearize the passive joints, which at first glance is somewhat surprising and nonintuitive. We show how this result can be used for the swing up control of the Acrobot [2].

\subsection{Collocated Linearization}

Consider the first equation (1)

$$
M_{11} \ddot{q}_{1}+M_{12} \ddot{q}_{2}+h_{1}+\phi_{1}=0
$$

The term $M_{11}$ is an invertible $\ell \times \ell$ matrix as a consequence of the uniform positive definiteness of the robot inertia matrix $M$ in (3). Therefore we may solve for $\ddot{q}_{1}$ in equation (6) as

$$
\ddot{q}_{1}=-M_{11}^{-1}\left(M_{12} \ddot{q}_{2}+h_{1}+\phi_{1}\right)
$$

and substitute the resulting expression (7) into (2) to obtain

$$
\bar{M}_{22} \ddot{q}_{2}+\bar{h}_{2}+\tilde{\phi}_{2}=\tau
$$


where the terms $\bar{M}_{22}, \bar{h}_{2}, \bar{\phi}_{2}$ are given by

$$
\begin{aligned}
\bar{M}_{22} & =M_{22}-M_{21} M_{11}^{-1} M_{12} \\
\bar{h}_{2} & =h_{2}-M_{21} M_{11}^{-1} h_{1} \\
\bar{\phi}_{2} & =\phi_{2}-M_{21} M_{11}^{-1} \phi_{1}
\end{aligned}
$$

As shown in [5] the $m \times m$ matrix $\bar{M}_{22}$ is itself symmetric and positive definite. To see this we note that a simple calculation [5] yields

$$
\bar{M}_{22}=T^{T} M T
$$

where $T$ is an $n \times m$ matrix defined by

$$
T=\left[\begin{array}{c}
-M_{11}^{-1} M_{12} \\
I_{m \times m}
\end{array}\right]
$$

with $I_{m \times m}$ the $m \times m$ identity matrix. Since $T$ has rank $m$ for all $q$ and $M$ is symmetric and positive definite, it follows that $\bar{M}_{22}$ is symmetric and positive definite.

A feedback linearizing controller can therefore be defined for equation (8) according to

$$
\tau=\bar{M}_{22} v_{2}+\bar{h}_{2}+\bar{\phi}_{2}
$$

where $v_{2} \in R^{m}$ is an additional control input yet to be defined. The complete system up to this point may be written as

$$
\begin{aligned}
M_{11} \ddot{q}_{1}+h_{1}+\phi_{1} & =-M_{12} v_{2} \\
\ddot{q}_{2} & =v_{2} \\
y_{2} & =q_{2}
\end{aligned}
$$

We see that the input/output system from $v_{2}$ to $y_{2}$ is linear and second order. The complete system therefore has $m$-vector relative degree $(2, \ldots, 2)^{T}[7]$ and the equation (12) represents the internal dynamics.

If $y_{2}^{d}=q_{2}^{d}(t)$ represents a desired trajectory for the active joints, then we may choose the additional control term $v_{2}$ as

$$
v_{2}=\ddot{q}_{2}^{d}+k_{d}\left(\dot{q}_{2}^{d}-\dot{q}_{2}\right)+k_{p}\left(q_{2}^{d}-q_{2}\right)
$$

where $k_{p}$ and $k_{d}$ are $m \times m$ diagonal matrices of positive gains. With state variables

$$
\begin{array}{ll}
z_{1}=q_{2}-q_{2}^{d} & z_{2}=\dot{q}_{2}-\dot{q}_{2}^{d} \\
\eta_{1}=q_{1} & \eta_{2}=\dot{q}_{1}
\end{array}
$$

and output error $\tilde{y}_{2}=y_{2}-y_{2}^{d}$, the complete closed loop system may be written as

$$
\begin{aligned}
\dot{z}_{1}= & z_{2} \\
\dot{z}_{2}= & -k_{p} z_{1}-k_{d} z_{2} \\
\dot{\eta}_{1}= & \eta_{2} \\
\dot{\eta}_{2}= & -M_{11}^{-1}\left(h_{1}+\phi_{1}\right) \\
& -M_{11}^{-1} M_{12}\left(\ddot{q}_{2}^{d}-k_{p} z_{1}-k_{d} z_{2}\right) \\
\tilde{y}_{2}= & z_{1}
\end{aligned}
$$

In matrix form we write this as

$$
\begin{aligned}
\dot{z} & =A z \\
\dot{\eta} & =w(z, \eta, t) \\
\tilde{y}_{2} & =C z
\end{aligned}
$$

where $z^{T}=\left(z_{1}^{T}, z_{2}^{T}\right), \eta=\left(\eta_{1}^{T}, \eta_{2}^{T}\right)$, the matrices $A$ and $C$ are given by

$$
A_{1}=\left[\begin{array}{cc}
0 & I_{m \times m} \\
-k_{p} & -k_{d}
\end{array}\right] \quad ; \quad C=\left[I_{m \times m}, 0\right]
$$

and the function $w(z, \eta, t)=$

$$
\left(-M_{11}^{-1}\left(h_{1}+\phi_{1}\right)-M_{11}^{-1} M_{12}\left(\ddot{q}_{2}^{\mathrm{d}}-k_{p} z_{1}-k_{d} z_{2}\right)\right)
$$

We see from (22) and (23) that the surface $z=0$ in state space defines an integral manifold for the system. Since $A$ is Hurwitz for positive values of gains in the matrices $k_{p}$ and $k_{d}$ this manifold is globally attractive. The dynamics on the manifold are given by

$$
\dot{\eta}=w(0, \eta, t)
$$

and define the zero dynamics [7] relative to the output $\tilde{y}=q_{2}-q_{2}^{d}$. We can state the following result whose proof can be found in [7] (see also [10]).

Theorem 1. Consider the system (22)-(24). Suppose that $w\left(0, \eta_{0}, t\right)=0$ for $t \geq 0$, i.e. $\left(0, \eta_{0}\right)$ is an equilibrium of the full system (22)-(24) and $\eta_{0}$ is an equilibrium of the zero dynamics (27). Suppose also that $A$ is a Hurwitz matrix. Then $\left(0, \eta_{0}\right)$ of the full system (22)-(24) is locally stable (respectively, locally asymptotically stable, unstable) if $\eta_{0}$ is locally stable (respectively, locally asymptotically stable, unstable) for the zero dynamics (27).

The point of this theorem is that the local stability properties of the full system may be determined based on the analysis of two reduced order systems, namely (22) and (27). An important point to note is that the Jacobian linearization of (23) may have eigenvalues on the imaginary axis and so not give sufficient information about the stability properties of the full nonlinear system. The proof of this result utilizes the Center Manifold Theorem and the reader is referred to [10] for details.

\subsection{Non-Collocated Input/Output Lin- earization}

In this section we show, under a condition regarding the degree of coupling between the active and passive 
joints, that instead of linearizing the active degrees of freedom $q_{2}$, we may linearize the passive degrees of freedom $q_{1}$ by nonlinear feedback. This result can be thought of as a combination of partial feedback linearization with the method of integrator backstepping.

To show this we consider the system (12)-(13)

$$
\begin{aligned}
M_{11} \ddot{q}_{1}+h_{1}+\phi_{1} & =-M_{12} v_{2} \\
\ddot{q}_{2} & =v_{2}
\end{aligned}
$$

Definition 1. The system (1)-(2), equivalently the system (28)-(29), is said to be Strongly Inertially Coupled if and only if

$$
\operatorname{rank}\left(M_{12}(q)\right)=\ell \quad \text { for all } q \in R^{n}
$$

This definition is essentially a controllability condition and ensures that the acceleration vector $v_{2}$ in (28) may be used as a control input to control the response of $q_{1}$ according to the recently developed method of Integrator Backstepping [9]. Note that Strong Inertial Coupling requires $m \geq \ell$, i.e. that the number of active degrees of freedom be at least as great as the number of passive degrees of freedom.

Under the assumption of Strong Inertial Coupling we may compute a pseudo-inverse $M_{12}^{\dagger}$ for $M_{12}$ according to

$$
M_{12}^{\dagger}=M_{12}^{T}\left(M_{12} M_{12}^{T}\right)^{-1}
$$

and define $v_{2}$ in (28) according to

$$
v_{2}=-M_{12}^{\dagger}\left(M_{11} v_{1}+h_{1}+\phi_{1}\right) \text {. }
$$

where $v_{1} \in R^{\ell}$ is an additional control input yet to be determined. With this choice for the control input $v_{2}$ the system becomes

$$
\begin{aligned}
& \ddot{q}_{1}=v_{1} \\
& \ddot{q}_{2}=-M_{12}^{\dagger}\left(M_{11} v_{1}+h_{1}+\phi_{1}\right)
\end{aligned}
$$

Thus we see that the passive degrees of freedom $q_{1}$ have been linearized and decoupled from the rest of the system and that the equation (34) describing the motion of the active joints now represents the internal dynamics of the system relative to an output equation $y_{1}=q_{1}$.

The actual control input $\tau$ is given by combining (11) and (32), after some algebra, as

$$
\tau=\tilde{M}_{21} v_{1}+\tilde{h}_{2}+\tilde{\phi}_{2}
$$

where

$$
\begin{aligned}
\tilde{M}_{21} & =M_{21}-M_{22} M_{12}^{\dagger} M_{11} \\
\tilde{h}_{2} & =h_{2}-M_{22} M_{12}^{\dagger} h_{1} \\
\tilde{\phi}_{2} & =\phi_{2}-M_{22} M_{12}^{\dagger} \phi_{1}
\end{aligned}
$$

A calculation similar to that previously given for $\bar{M}_{22}$ shows that $\tilde{M}_{21}$ has full rank $\ell$, since we may write

$$
\left[\begin{array}{ll}
M_{11} & M_{12} \\
M_{21} & M_{22}
\end{array}\right]\left[\begin{array}{c}
I_{\ell \times \ell} \\
-M_{12}^{\dagger} M_{11}
\end{array}\right]=\left[\begin{array}{c}
0 \\
\tilde{M}_{21}
\end{array}\right]
$$

where $I_{\ell \times \ell}$ is the $\ell \times \ell$ identity matrix. Since the inertia matrix is invertible and the matrix

$$
\left[\begin{array}{c}
I_{\ell \times \ell} \\
-M_{12}^{\dagger} M_{11}
\end{array}\right]
$$

has full column rank $\ell$, it follows that the $\tilde{M}_{21}$ has rank $\ell$ and thus the control is well defined.

If $q_{1}^{d}(t)$ now represents a desired trajectory for the passive joints, we may choose the additional control term $v_{1}$ as

$$
v_{1}=\ddot{q}_{1}^{d}+k_{d}\left(\dot{q}_{1}^{d}-\dot{q}_{1}\right)+k_{p}\left(q_{1}^{d}-q_{1}\right)
$$

where $k_{p}$ and $k_{d}$ are $\ell \times \ell$ matrices of positive gains. With state variables

$$
\begin{array}{ll}
\eta_{1}=q_{1}-q_{1}^{d} & \eta_{2}=\dot{q}_{1}-\dot{q}_{1}^{d} \\
z_{1}=q_{2} & z_{2}=\dot{q}_{2}
\end{array}
$$

and output error $\tilde{y}_{1}=q_{1}-q_{1}^{d}$ the closed loop system may be written as

$$
\begin{aligned}
& \dot{\eta}_{1}=\eta_{2} \\
& \dot{\eta}_{2}=-k_{p} \eta_{1}-k_{d} \eta_{2} \\
& \dot{z}_{1}=z_{2} \\
& \dot{z}_{2}=-M_{12}^{\dagger}\left(h_{1}+\phi_{1}\right) \\
& \quad-M_{12}^{\dagger} M_{11}\left(\ddot{q}_{1}^{d}-k_{p} \eta_{1}-k_{d} \eta_{2}\right) \\
& \tilde{y}_{1}=\eta_{1}
\end{aligned}
$$

In matrix form we write this as

$$
\begin{aligned}
\dot{\eta} & =A \eta \\
\dot{z} & =s(\eta, z, t) \\
\tilde{y}_{1} & =C \eta
\end{aligned}
$$

where $\eta^{T}=\left(\eta_{1}^{T}, \eta_{2}^{T}\right), z^{T}=\left(z_{1}^{T}, z_{2}^{T}\right)$, the matrices $A$ and $C$ are given by

$$
A=\left[\begin{array}{cc}
0 & I_{\ell \times \ell} \\
-k_{p} & -k_{d}
\end{array}\right] ; \quad C=\left[I_{\ell \times \ell}, 0\right]
$$

and the function $s(\eta, z, t)=$

$$
\left(\begin{array}{c}
z_{2} \\
-M_{12}^{\dagger}\left(h_{1}+\phi_{1}\right)-M_{12}^{\dagger} M_{11}\left(\ddot{q}_{1}^{d}-k_{p} \eta_{1}-k_{d} \eta_{2}\right)
\end{array}\right)
$$


We see that the surface $\eta=0$ in state space defines a globally attractive integral manifold for the system and that the expression

$$
\dot{z}=s(0, z, t)
$$

defines the zero dynamics relative to the output $\tilde{y}_{1}=$ $\eta_{1}$. Theorem 1 above applies to this system as well, i.e., an equilibrium $\left(0, z_{0}\right)$ of the full system (45)-(47) is locally stable (respectively, locally asymptotically stable, unstable) if $A$ is Hurwitz and if the equilibrium $z_{0}$ of the zero dynamics $(50)$ is locally stable (respectively, locally asymptotically stable, unstable).

\section{Examples}

In this section we give examples of both the collocated and non-collocated partial feedback linearization control. We treat the so-called swing up control of the Acrobot[15], a two-link robot with an actuator at the elbow but no actuator at the shoulder. Both the collocated and non-collocated linearization results are illustrated.

\subsection{Swing-Up Control of the Acrobot}

The swing up control problem for the Acrobot (shown in Figure 3) is to swing the Acrobot from its stable downward equilibrium to its unstable inverted position and balance it about the vertical. The equa-

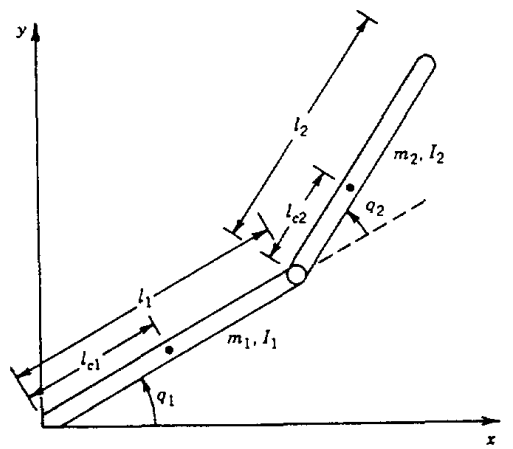

Figure 3: The Acrobot

tions of motion for the Acrobot are

$$
\begin{aligned}
& m_{11} \ddot{q}_{1}+m_{12} \ddot{q}_{2}+h_{1}+\phi_{1}=0 \\
& m_{21} \ddot{q}_{1}+m_{22} \ddot{q}_{2}+h_{2}+\phi_{2}=\tau_{2}
\end{aligned}
$$

where

$$
m_{11}=m_{1} \ell_{c 1}^{2}+m_{2}\left(\ell_{1}^{2}+\ell_{c 2}^{2}+2 \ell_{1} \ell_{c 2} \cos \left(q_{2}\right)\right)+I_{1}+I_{2}
$$$$
m_{22}=m_{2} \ell_{c 2}^{2}+I_{2}
$$

$$
\begin{aligned}
m_{12} & =m_{21}=m_{2}\left(\ell_{c 2}^{2}+\ell_{1} \ell_{c 2} \cos \left(q_{2}\right)\right)+I_{2} \\
h_{1} & =-m_{2} \ell_{1} \ell_{c 2} \sin \left(q_{2}\right) \dot{q}_{2}^{2}-2 m_{2} \ell_{1} \ell_{c 2} \sin \left(q_{2}\right) \dot{q}_{2} \dot{q}_{1} \\
h_{2} & =m_{2} \ell_{1} \ell_{c 2} \sin \left(q_{2}\right) \dot{q}_{1}^{2} \\
\phi_{1} & =\left(m_{1} \ell_{c 1}+m_{2} \ell_{1}\right) g \cos \left(q_{1}\right)+m_{2} \ell_{c 2} g \cos \left(q_{1}+q_{2}\right) \\
\phi_{2} & =m_{2} \ell_{c 2} g \cos \left(q_{1}+q_{2}\right)
\end{aligned}
$$

The simulations to follow were written in Simnon using the parameters in Table 1 below.

\begin{tabular}{|l|l|l|l|l|l|l|l|l|}
\hline$m_{1}$ & $m_{2}$ & $\ell_{1}$ & $\ell_{2}$ & $\ell_{c_{1}}$ & $\ell_{c_{2}}$ & $I_{1}$ & $I_{2}$ & $g$ \\
\hline 1 & 1 & 1 & 1 & 0.5 & 0.5 & 0.2 & 1.0 & 9.8 \\
\hline
\end{tabular}

Table 1: Parameters of the Simulated Acrobot

\subsection{Collocated Linearization}

In order to swing up the robot from the vertically downward configuration $q_{1}=-\pi / 2, q_{2}=0$ to the inverted configuration $q_{1}=+\pi / 2, q_{2}=0$, we apply the collocated partial feedback linearization control (11) with the outer loop term given by (15). The motion of the second link, in the $z$-coordinates, is then just the response of a second-order linear system. This motion will excite the internal dynamics which produces the motion of the first link. The crucial step in this procedure is then the determination of the reference input $q_{2}^{d}$ for the second link.

The basic idea behind our swingup strategy is to swing the second link between fixed values $\pm \alpha$ in order to pump energy into the system and then to schedule the transition of the second link between these two values $\pm \alpha$ "in phase" with the motion of the first link in such a way that the amplitude of the swing of the first link increases with each swing (See [15] for details). We do this by making the reference $q_{2}^{d}$ for link 2 a feedback function of the velocity $\dot{q}_{1}$ of link 1 as follows:

$$
q_{2}^{d}=2 \alpha / \pi \arctan \left(\dot{q}_{1}\right)
$$

as shown in Figure 4.

It is interesting and important to note that our choice of reference command to link 2 as a pure feedback function of $\dot{q}_{1}$ renders the system autonomous. Therefore the zero dynamics evolve on an invariant manifold in state space.

Substituting (53) into (27) yields, after a straightforward calculation, the following expression for the zero dynamics: 


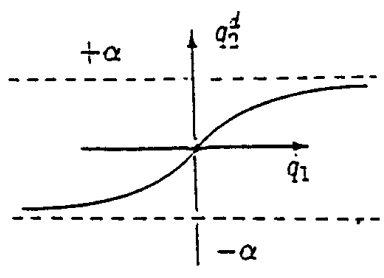

Figure 4: Arctangent Function

$$
\begin{aligned}
& \frac{m_{12}\left(q_{2}^{d}\right)}{1+\dot{q}_{1}^{2}} \frac{d^{3} q_{1}}{d t^{3}}+m_{11}\left(q_{2}^{d}\right) \ddot{q}_{1}-2 \frac{m_{12}\left(q_{2}^{d}\right) \dot{q}_{1}}{\left(1+\dot{q}_{1}^{2}\right)^{2}} \ddot{q}_{1}^{2} \\
& +h_{1}\left(\dot{q}_{1}, q_{2}^{d}, \dot{q}_{2}^{d}\right)+\phi\left(q_{1}, q_{2}^{d}\right)=0
\end{aligned}
$$

Remarks: We see that the zero dynamics for this system is an autonomous third order nonlinear system. This can be explained as follows. Using the expression (53) for the reference command $q_{2}^{d}$ in the outer loop control (15) means that the feedforward terms $\dot{q}_{2}^{d}$ and $\ddot{q}_{2}^{d}$ contain the acceleration and jerk, respectively, of link 1 and thus the order of the system is increased by one. Figure 5 shows the response of the zero dynamics (54). We see that the equilibrium $q_{1}=-\pi / 2$ is unstable. The response is plotted modulo $2 \pi$ which is the reason for the apparent jumps between 10 and 14 seconds where the angle reaches $2 \pi$.
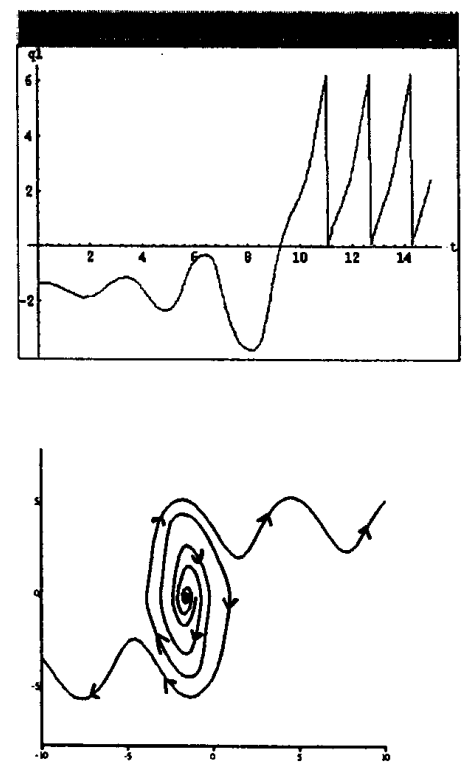

Figure 5: Response of the Zero Dynamics (Initial Condition $\left.q_{1}(0)=-1.35\right)$ and a Portion of the Phase Portrait
We note, however, that the control law 15 , is not realizable using only position and velocity measurements. In order to obtain a realizable control input, therefore, we will use, instead of (15), the control

$$
v_{2}=k_{p}\left(q_{2}^{d}-q_{2}\right)-k_{d} \dot{q}_{2},
$$

i.e., (15) without the feedforward terms $\dot{q}_{2}^{d}$ and $\ddot{q}_{2}^{d}$. The control law (55) requires only position and velocity measurements to implement. The price we pay for the simplified outer loop control (55) is that the $z$-coordinates are no longer decoupled from the $\eta$ coordinates in (22) $-(23)$ and the manifold $z=0$ is no longer invariant. Figure 6 show the response of link 1 for the actual system using the outer loop control (55). Note that the response is quite similar to the response of the ideal zero dynamics (54). As the gains $k_{p}$ and $k_{d}$ are increased in (55) the response of the system becomes nearly the same as the response predicted by the ideal case.

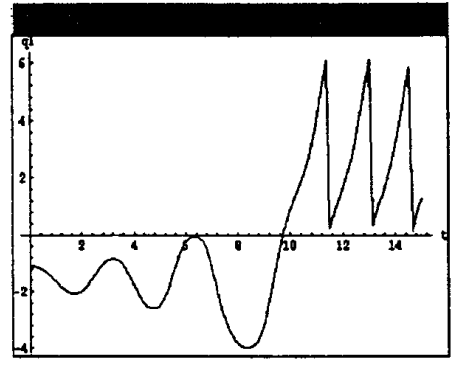

Figure 6: Actual Response of Link 1 Using Collocated Control (Initial Condition $q_{1}(0)=-1.1$ )

The swing up motion is now accomplished by combining the above partial feedback linearization control with a Linear Quadratic Regulator. Control is switched to the linear regulator to balance the Acrobot about the vertical when the Acrobot enters the basin of attraction of the linear regulator. See [15] for details of the design of the linear regulator. Figure 7 shows a swing up motion using the reference $q_{2}^{d}$ for $q_{2}$ given by (53).

\subsection{Non-Collocated Linearization}

We can also develop an interesting swing up control for the Acrobot using the non-collocated linearization result. The condition of Strong Inertial Coupling for the Acrobot requires that $m_{12}$ be non-zero over the entire configuration space, i.e,

$$
m_{12}=m_{2}\left(\ell_{c 2}^{2}+\ell_{1} \ell_{c 2} \cos \left(q_{2}\right)\right)+I_{2} \neq 0 .
$$




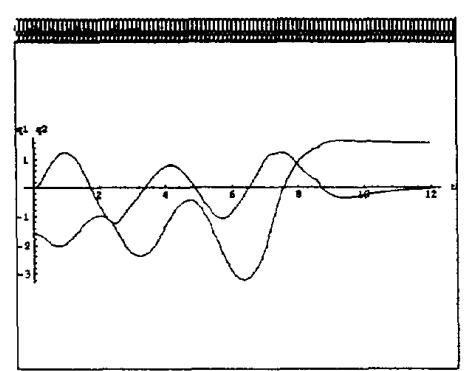

Figure 7: Swingup and Balance of The Acrobot Using Collocated Control

This imposes a constraint on the inertia parameters of the robot, namely that $I_{2}>m_{2} \ell_{c 2}\left(\ell_{1}-\ell_{c 2}\right)$. Using the parameters from Table 1 we see that the Strong Inertial Coupling condition is satisfied since $m_{12}=1.25+0.5 \cos \left(q_{2}\right)$. Therefore we may use the control law (35).

Since we are interested in the swing up control problem, we consider the case $q_{1}^{d}=\pi / 2$. We then choose the outer loop term as

$$
v_{1}=k_{p}\left(q_{1}^{d}-q_{1}\right)-k_{d} \dot{q}_{1}
$$

and the system is once again autonomous. Substituting $q_{1}^{d}=\pi / 2, \dot{q}_{1}^{d}=0=\ddot{q}_{1}^{d}$ into the equation (50) and using the original description of the system (51) yields

$$
\begin{aligned}
& \left(m_{2} \ell_{c 2}^{2}+m_{2} \ell_{1} \ell_{c 2} \cos \left(q_{2}\right)+I_{2}\right) \ddot{q}_{2} \\
& -\quad m_{2} \ell_{1} \ell_{c 2} \sin \left(q_{2}\right) \dot{q}_{2}^{2}-m_{2} \ell_{c 2} g \sin \left(q_{2}\right)=0
\end{aligned}
$$

as the expression for the zero dynamics. The system (57), considered as a dynamical system on the cylinder, has two equilibrium points $p_{1}=(0,0)^{T}$, which is a saddle, and $p_{2}=(\pi, 0)^{T}$, which is a center. With the parameters from Table 1 the phase portrait of the zero dynamics (57) is shown in Figure 8. It follows that, for a range of initial conditions, $z(0)=z_{0}, \eta(0)=\eta_{0}$, the state $\eta(t)=\left(q_{1}-q_{1}^{d}, \dot{q}_{1}\right)^{T}$ converges exponentially to zero, while the state $z(t)=\left(q_{2}, \dot{q}_{2}\right)^{T}$ converges to a trajectory of the system (57).

The particular trajectory of the zero dynamics that the response of the system converges to will depend both on the outer loop gains and on the initial conditions. Figure 9 shows a successful swing up and balance using this approach where the control again switches to a Linear, Quadratic Regulator to balance the Acrobot when the trajectory nears the vertical position.

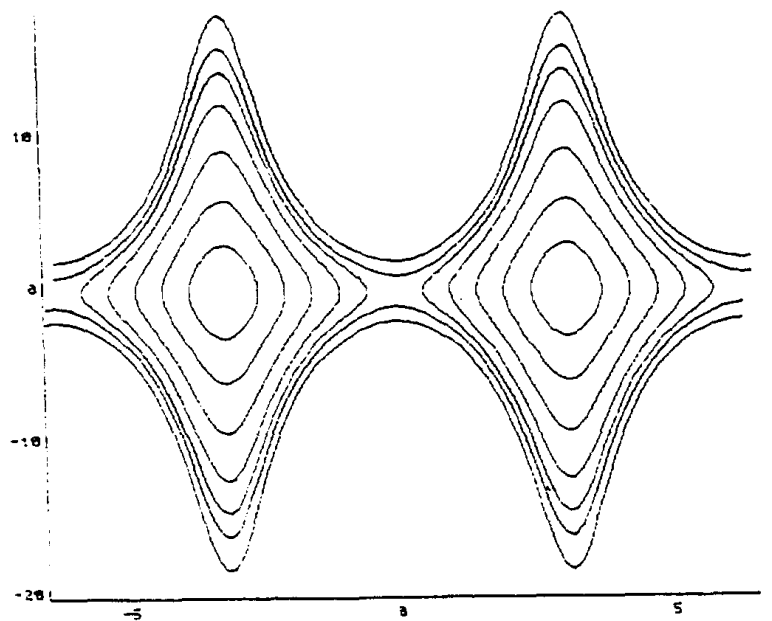

Figure 8: Phase Portrait of the Zero Dynamics

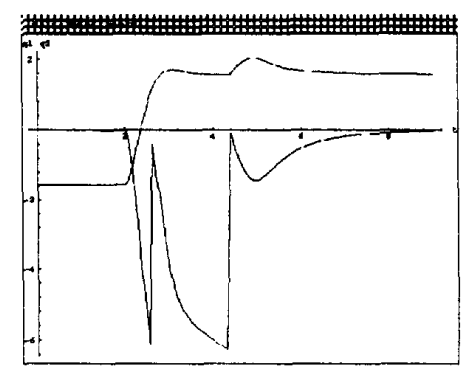

Figure 9: Swing Up and Balance of the Acrobot Using Noncollocated Control

\section{Conclusions}

In this paper we have shown that the methods of partial feedback linearization and integrator backstepping provide effective design tools for the control of a class of underactuated mechanical systems. We have shown that the analysis of the resulting internal or zero dynamics is crucial to an understanding of the behavior of the overall system.

Research into the control of this class of robotic systems is just beginning and there are a number of research problems that remain to be addressed. It would be desirable to develop a theory of robust and adaptive control for these systems. A major impediment to progress in this area is that the reduced order system (8) is not linearly parametrizable, in general. This means that standard adaptive control and backstepping methods are not applicable. A second research problem is to further analyze the zero dynamics for classes of problems and applications. The zero dynamics are determined by the particular control laws used and vary greatly from system to system. 
Although space restrictions did not permit in this paper, it is also possible to derive partial feedback linearization control laws directly in task space coordinates as opposed to the joint space linearization considered here. In this case, singularities are of major concern and methods of controlling such system through singularities is a difficult and open problem. The singularities that arise in task space linearization of underactuated systems will depend, in general, on both the kinematic and dynamic parameters of the system in contrast to the case of fully actuated system where the singularities depend only on the kinematic parameters [4]. This fact greatly impacts both the control and the motion planning problems for underactuated systems[11].

\section{References}

[1] Arai, H. and Tachi, S. "Position Control of a Manipulator with Passive Joints Using Dynamic Coupling," IEEE Trans. Robotics and Automation, Vol. 7, No. 4, pp. 528-534, Aug. 1991.

[2] Bortoff, S.A., Pseudolinearization using Spline Functions with Application to the Acrobot, Ph.D. Thesis, Dept. of Electrical and Computer Engineering, University of Illinois at UrbanaChampaign, 1992.

[3] Bortoff, S., and Spong, M.W., "Pseudolinearization of the Acrobot Using Spline Functions," IEEE Conf. on Decision and Control, Tucson, AZ, pp. 593-598, Dec. 1992.

[4] Dubowsky, S., and Papadopoulos, E., "The Kinematics, Dynamics, and Control of Free-Flying and Free-Floating Space Robotic Systems," IEEE Trans. on Robotics and Automation, Vol. 9, NO. $5,1993$.

[5] Gu, Y-L, and Xu, Y., "A Normal Form Augmentation Approach to Adaptive Control of Space Robot Systems," Proc. IEEE Int. Conf. on Robotics and Automation, pp. 731-737, Atlanta, GA, May, 1993.

[6] J. Hauser and R. M. Murray. Nonlinear controllers for non-integrable systems: the acrobot example. In Proc. American Control Conference, 1990.

[7] Isidori, A., Nonlinear Control Systems, 2nd Edition, Springer-Verlag, Berlin, 1989.
[8] Jain, A., and Rodriguez, G., "An Analysis of the Kinematics and Dynamics of Underactuated Manipulators," IEEE Trans. on Robotics and Automation, Vol. 9, No. 4, pp. 411-422, Aug. 1993.

[9] Kokotović, P.V., Krstić, M., and Kanellakopoulos, I., "Backstepping to Passivity: Recursive Design of Adaptive Systems," Proc. IEEE Conf. on De cision and Control, pp. 3276-3280, Tucson, AZ, Dec. 1992.

[10] Khalil, H., Nonlinear Systems, Macmillan Publishing Co., New York, 1992.

[11] Papadopoulos, E., and Dubowsky, S., "Coordinated Manipulator/Spacecraft Motion Control for Space Robotic Systems," Proc. IEEE IntI. Conf. on Robotics and Automation, Sacramento, CA, April, 1991.

[12] Papadopoulos, E., and Dubowsky, S., "Dynamic Singularities in Free-Floating Space Manipulators," ASME J. Dynamical Systems, Measurement, and Control, Vol. 115, March, 1993.

[13] Saito, F., Fukuda, T., and Arai, F., "Swing and Iocomotion Control for Two-Link Brachiation Robot," Proc. 1993 IEEE Int. Conf. on Robotics and Automation, pp. 719-724, Atlanta, GA, 1993.

[14] Spong, M.W., "Modeling and Control of Elastic Joint Robots", ASME J. Dyn. Systems, Meas. and Control, Vol. 109, pp. 310-319, December, 1987.

[15] Spong, M.W., "Swing Up Control of the Acrobot," 1994 IEEE Int. Conf. on Robotics and Automation, pp. 2356-2361, San Diego, CA, May, 1994.

[16] Spong, M.W., and Vidyasagar, M., Robot Dynamics and Control, John Wiley \& Sons, Inc., New York, 1989.

[17] Takashima, S., "Control of a Gymnast on a High Bar," IEEE Int'l Workshop on Intelligent Robots and Systems, IROS'91, pp. 1424-1429, Osaka, Japan, Nov. 1991.

[18] Wiklund, M., Kristenson, A., and Astrom, K.J., "A New Strategy for Swinging up an Inverted Pendulum," Proc. IFAC Symposium, Sydney, Australia, 1993. 